\title{
Water Quality of Selected Fishing Beaches of Lake Victoria Kenyan Gulf
}

\author{
Paul S. Orina ${ }^{*}$, David M. Onyango², Henry Lungayia ${ }^{3}$, Andrew Oduor ${ }^{2}$, Anthony W. Sifuna ${ }^{3}$, \\ Petronila Otuya ${ }^{4}$, Rosemary A. Owigar ${ }^{5}$, Chrispin B. Kowenje ${ }^{2}$, Sheban M. Hinzano ${ }^{1}$
}

\author{
${ }^{1}$ Kegati Aquaculture Centre, Kenya Marine and Fisheries Research Institute, Kisii, Kenya \\ ${ }^{2}$ Department of Zoology, School of Physical and Biological Sciences, Maseno University, Maseno, Kenya \\ ${ }^{3}$ Department of Biological Sciences, School of Biological and Physical Sciences, Masinde Muliro University of Science and \\ Technology, Kakamega, Kenya \\ ${ }^{4}$ Department of Social Sciences, School of Health Sciences, St. Paul's University, Limuru, Kenya \\ ${ }^{5}$ Department of Geography, School of Environmental Sciences, Maseno University, Maseno, Kenya \\ Email: *paulorina@gmail.com
}

How to cite this paper: Orina, P.S., Onyango, D.M., Lungayia, H., Oduor, A., Sifuna, A.W., Otuya, P., Owigar, R.A., Kowenje, C.B. and Hinzano, S.M. (2020) Water Quality of Selected Fishing Beaches of Lake Victoria Kenyan Gulf. Open Journal of Ecology, 10, 22-35.

https://doi.org/10.4236/oje.2020.101002

Received: November 5, 2019

Accepted: January 13, 2020

Published: January 16, 2020

Copyright $\odot 2020$ by author(s) and Scientific Research Publishing Inc. This work is licensed under the Creative Commons Attribution International License (CC BY 4.0).

http://creativecommons.org/licenses/by/4.0/ (c) (i) Open Access

\begin{abstract}
Lake Victoria the second largest fresh water body in the world located in East Africa is a shared resource between Kenya, Tanzania and Uganda and enjoys a wide range of streams and rivers from as far as Burundi and Rwanda. The lake has environmentally undergone physical, chemical and biological changes in the last four decades, particularly rise in its trophic condition and decline in oxygen level, which affects the water quality and fish population dynamics. This study therefore set out to determine the quality of water in selected fishing beaches of Lake Victoria, Kenya with a view to report the possible pollution levels. $\mathrm{pH}$ was highest at Nyamasari and Kotieno $(9.3 \pm$ $0.1)$ and lowest at Nyachebe and Kichinjio $(7.08 \pm 0.1)$ whereas temperature was highest at Nyamasari $\left(29.5^{\circ} \mathrm{C} \pm 0.0^{\circ} \mathrm{C}\right)$ and lowest at Kichinjio $\left(23.4^{\circ} \mathrm{C} \pm\right.$ $\left.0.2^{\circ} \mathrm{C}\right)$. DO was highest at Kotieno $(10.3 \pm 0.2 \mathrm{mgL})$ and lowest at Seka $(2.4 \pm$ $0.1 \mathrm{mgL})$. Turbidity was highest at Uyoga (125.5 $\pm 0.90 \mathrm{NTU})$ and lowest at Osieko $(2.7 \pm 0.1 \mathrm{NTU})$. Ammoniacal nitrogen was highest at Dunga (1278.3 $\left.\pm 0.8 \mu \mathrm{g} \cdot \mathrm{l}^{-1}\right)$ and lowest at Nyamasari (12.4 \pm 0.8$)$, all a factor of human activities, lake substratum and effluents from rivers and surface runoff. All parameters assessed showed significant differences across sampling sites and depth except $\mathrm{pH}$ which did not vary significantly with distance from lakeshore. Further, all parameters did not show a clear pattern with respect to distance from the lakeshore possibly due to adequate mixing in the gulf. There is a need for further water quality monitoring by seasons to inform policy decisions towards sustainable lake exploitation.
\end{abstract}

\section{Keywords}

Water Quality, Pollution, Human Activities, Sampling Sites, Lakeshore 


\section{Introduction}

Lake Victoria the second largest fresh water body in the world located in East Africa is a shared resource between Kenya, Tanzania and Uganda and enjoys a wide range of streams and rivers from as far as Burundi and Rwanda [1] [2]. The Kenyan waters of L. Victoria lie just south of the equator between 004'S - 0032'S and $34^{\circ} 13^{\prime} \mathrm{E}-34^{\circ} 52^{\prime} \mathrm{E}$, and cover an area of $3600 \mathrm{~km}^{2}$ of which $1400 \mathrm{~km}^{2}$ is the Winam Gulf [3]. Unlike Uganda and Tanzania, the Kenyan waters shoreline is dominated by the Nyanza Gulf an important economic and ecological water front to an estimated 3 million people settled along the gulf shores. The shared vision and strategic frame work for Lake Victoria basin muted in 2003 is to have a prosperous population living in a healthy and sustainably managed environment providing equitable opportunity and benefits [4]. It is documented that human society has over time used freshwater from rivers, lakes, groundwater and wetlands for many different rural, peri-urban and urban agricultural and industrial activities, but in doing so has overlooked its value in supporting ecosystems. Freshwater is vital to human life and societal well-being, and thus its utilization for consumption, irrigation and transport has long taken precedence over other commodities and services provided by freshwater ecosystems [5]. However, this has not been possible due to frequent pollution of the lake thus affecting human health and fish environment [6]. Lake Victoria has environmentally undergone physical, chemical and biological changes in the last four decades, particularly rise in its trophic condition and decline in oxygen level, which affects the water quality and fish population dynamics [6] [7]. This is attributed to increased nutrient loading from both atmospheric deposition and surface run-off both being factors of rising human population and their associated anthropogenic activities resulting in growing changes in the lake's phytoplankton community [8]. The pollution impact by municipal and industrial discharge is visible in some of the rivers discharging into the largest East African lake as well as the shoreline mainly along shallow areas of Kenya's Winam Gulf in Kisumu, Tanzania's Mwanzaarea and Uganda's Inner Murchison Bay as well as Jinja catchment [9] [10] [11] [12]. A number of factors have been listed to affect the quality of Lake Victoria waters among them being rapid population growth, increased agriculture, urbanization, industrial activities, poverty in rural and peri-urban areas, and uncontrolled dumping of waste among others despite existing environmental policies [13] [14]. According to [15], forests are being cut down, soils are eroded, wetlands are drained, channels are silted accelerating lake water pollution from non-point sources (NPS) further reducing access to already limited freshwater for human use. According to [16], the increase in cereal demand in Africa by 2025 estimated at $65 \%$ resulting from rising human population will require more water for irrigation. The anticipated threefold increase in water demand by Africa will emanate from municipal and domestic sectors which will account for an estimated 320 billion cubic meters while competition with other water using sectors, notably agriculture will account for 92 
billion cubic meters [17]. In this regard, [18] states that the amount of water available per person in Africa is far below the global average and is declining with annual per capita availability of 4000 cubic meters compared to a global average of 6500 cubic meters. The science challenges dealing with marine international waters such as L. Victoria at a trans-boundary, regional and global level include multiple stressors such as eutrophication, overfishing, habitat destruction, pollution, harmful algal blooms and the movement of opportunistic invasive species [19].

Lake shore development has also been identified as one of the factors that impact critical ecosystem processes and ecological features of the adjacent lake [20]. Residential development for instance has been associated with increased siltation and fish exploitation rates, and reduction of habitat complexity which collectively affect fish populations and growth more so of species that use the habitats at a given development stage in their life cycle. [21] reported a negative correlation between size-specific growth rate and degree of residential development in bluegill sunfish. In the same study, bluegill in highly developed lake shores were found to be less productive (2.3 times less) as compared to those in lowly developed lake shores. Despite the enormous knowledge of all these factors affecting water quality, there are no mechanisms for assessing the impact of the resultant loads on the surface and subsurface water, and ultimately the potential of the lake to support productive aquatic life, thus the reason for the current study.

The current study therefore set-out to determine the quality of water in selected fishing beaches of Lake Victoria, Kenya with a view to report the possible pollution levels within the waters that would contribute directly or indirectly to fisheries and fish biology in the region.

\section{Materials and Methods}

\subsection{Study Sites}

The study area comprised of near-shore shallow waters at fish landing beaches mainly in the Nyanza Gulf and selected parts of the main lake, Kenya side (Figure 1). The environmental characteristics including aquatic vegetation cover, human settlement and activities at each beach also referred to as site were considered as well as natural conditions influencing water quality and their effect on the quality of harvested fish. In almost all the sites, landed fish were cleaned with water obtained from near the shores of the lake. The choice of the sampling site were as a result of hygienic levels of by-catch and processed fish waste disposal, human settlement associated anthropogenic activities and existing records on Lake Victoria pointing at pollution and eutrophication. A total of 12 fish landing beaches were investigated within the wet and dry seasons (Figure 1; Table 1). Six beaches (sites 1 - 6) were sampled in September 2015 and the other six (sites 7 - 12) in November 2015 between 09:00 hrs and 15:30 hrs. The location of each sampling site was determined using a Geographical Positioning System (Garmin 


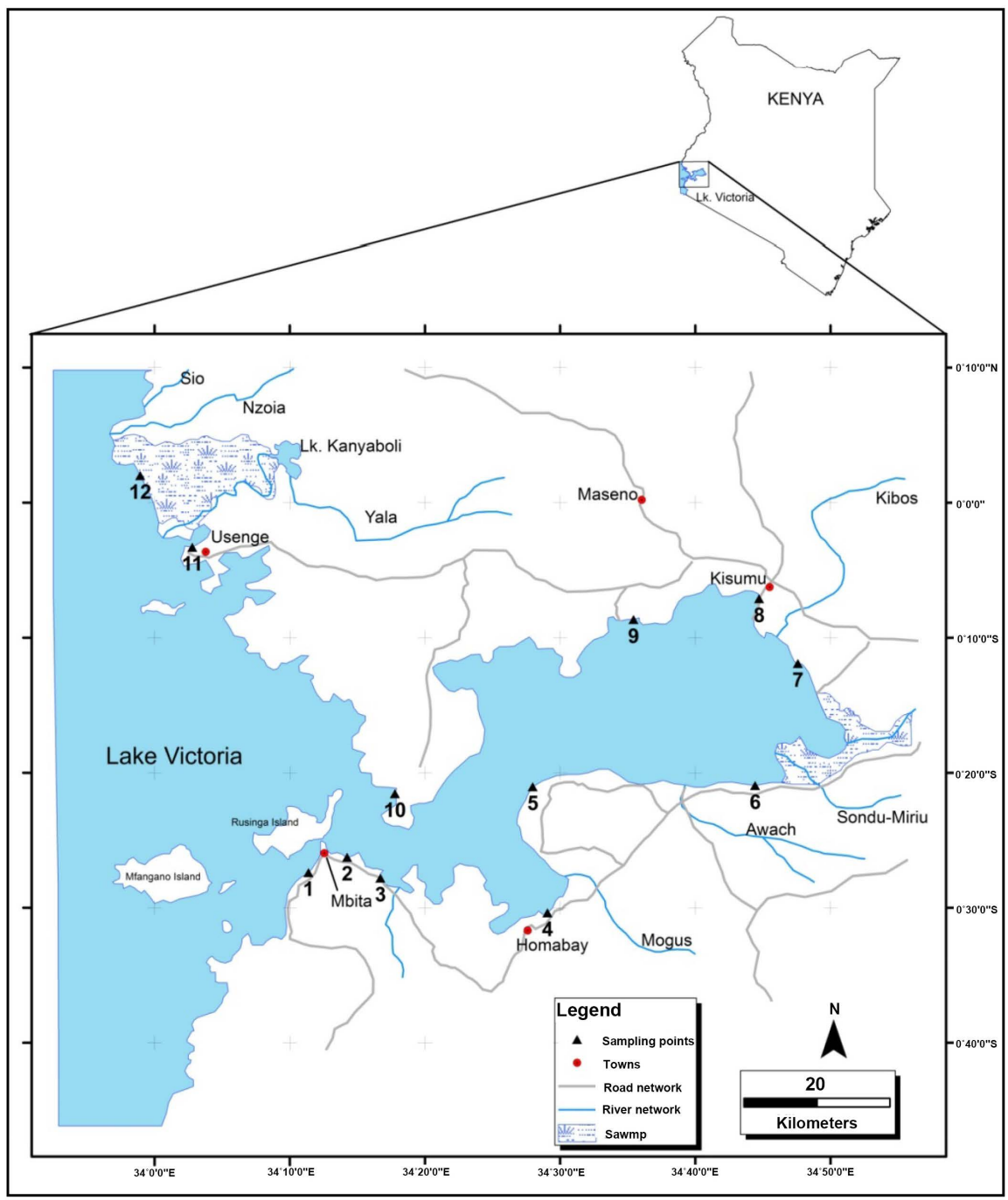

Figure 1. Map of the study area showing the position of the sampling sites (points (1 12)) on Lake Victoria.

tino 130). Three (triplicate) measurements and water samples were taken at each sampling site, one set immediately at the shoreline 2 - 4 meters offshore $(\leq 1 \mathrm{~m}$ depth) and a second set $100-200 \mathrm{~m}$ ( $\leq 5 \mathrm{~m}$ depth). In situ measurements of water temperature, $\mathrm{pH}$, electrical conductivity and turbidity were taken with a Hydrolab (Hand-held Water Quality Meter WQC-24) and total dissolved solids TDS with an Oyster ${ }^{\mathrm{TM}}$ meter. Secchi depth was estimated with a $20 \mathrm{~cm}$ diameter black and white Secchi disc.

\subsection{Water Sampling and Analysis}

Water samples were collected from $0.5 \mathrm{~m}$ below the surface with polyethylene bottles, stored at $4^{\circ} \mathrm{C}$ in a cooler box and later transferred to a refrigerator $\left(-20^{\circ} \mathrm{C}\right)$ in the laboratory until analysis. Prior to determination, water samples were brought to room temperature and filtered through $0.45 \mu \mathrm{m}$ pore size filters and immediately frozen at $-20^{\circ} \mathrm{C}$ in readiness for analysis. The water samples 
Table 1. Description of the 12 sampling sites on Lake Victoria (Kenya part).

\begin{tabular}{|c|c|c|c|c|}
\hline \multirow[t]{2}{*}{ Code } & \multirow[t]{2}{*}{ Site name } & \multicolumn{2}{|c|}{ GPS position } & \multirow[t]{2}{*}{ Characteristics of beach and activities } \\
\hline & & Latitude & Longitude & \\
\hline 1 & Nyachebe & $0^{\circ} 2732$ 'S & $34^{\circ} 118^{\prime} \mathrm{E}$ & Busy fish landings, water used for laundry, cleaning utensils, bathing. \\
\hline 2 & Uyoga & $0^{\circ} 267^{\prime} \mathrm{S}$ & $34^{\circ} 14^{\prime} 11^{\prime} \mathrm{E}$ & Small urban centre, laundry, bathing. \\
\hline 3 & Luanda Nyamasari & $0^{\circ} 2739^{\prime} \mathrm{S}$ & $34^{\circ} 1634^{\prime} \mathrm{E}$ & Rural settlement, laundry, livestock, water hyacinth mats near shoreline. \\
\hline 4 & Ngegu & $0^{\circ} 3022$ 'S & $34^{\circ} 2859^{\prime} \mathrm{E}$ & $\begin{array}{l}\text { Busy landing, laundry, cleaning utensils, domestic abstraction, with } \\
\text { many birds. }\end{array}$ \\
\hline 5 & Mainunga & 0211 's & $34^{\circ} 27^{\prime} 47^{\prime} \mathrm{E}$ & Busy landing, laundry, cleaning utensils, domestic abstraction. \\
\hline 6 & Seka & $0^{\circ} 211$ 'S & $34^{\circ} 44^{\prime} 19^{\prime} \mathrm{E}$ & Busy landing, muddy beach, water hyacinth mats cover up to shoreline. \\
\hline 7 & Dunga & $0^{\circ} 1152$ 'S & $34^{\circ} 4722^{\prime} \mathrm{E}$ & $\begin{array}{l}\text { Urban centre, eatery on beach, busy landing, passenger boats, laundry, } \\
\text { bathing, water hyacinth mats in vicinity. }\end{array}$ \\
\hline 8 & Kichinjio & $0^{\circ} 75^{\prime} \mathrm{S}$ & $34^{\circ} 4438^{\prime \prime} \mathrm{E}$ & $\begin{array}{l}\text { Urban centre, several eateries on beach, car wash inside the water, busy } \\
\text { landing, and passenger boats, laundry, cleaning utensils, swimming. }\end{array}$ \\
\hline 9 & Kagwel & $0^{\circ} 836^{\prime} \mathrm{S}$ & $34^{\circ} 35^{\prime} 17^{\prime \prime} \mathrm{E}$ & $\begin{array}{l}\text { Laundry, cleaning utensils, domestic abstraction, and water hyacinth } \\
\text { mats near shoreline. }\end{array}$ \\
\hline 10 & Luanda Kotieno & $0^{\circ} 2133^{\prime} \mathrm{S}$ & $34^{\circ} 179^{\prime \prime} \mathrm{E}$ & Urban centre, busy landing, passenger boats, laundry. \\
\hline 11 & Usenge & $0^{\circ} 317^{\prime} \mathrm{S}$ & $34^{\circ} 239^{\prime} \mathrm{E}$ & Urban centre, busy landing, passenger boats, laundry. \\
\hline 12 & Osieko & $0^{\circ} 157^{\prime \prime} \mathrm{N}$ & $34^{\circ} 5850^{\prime} \mathrm{E}$ & $\begin{array}{l}\text { Urban centre, busy landing, passenger boats, laundry, cleaning utensils, } \\
\text { sand-harvesting. }\end{array}$ \\
\hline
\end{tabular}

were subjected to various analyses using methods selected from [22] They included spectrophotometric methods for phosphate-phosphorus $\mathrm{PO}_{4}-\mathrm{P}$ (soluble reactive phosphorus SRP, ascorbic acid) and Ammonium-nitrogen $\mathrm{NH}_{4}-\mathrm{N}$ (Manual phenate, indophenol-blue). Total suspended solids (TSS) were estimated from residue filtered on GFC filters and dried at $103^{\circ} \mathrm{C}-105^{\circ} \mathrm{C}$. Chlorophyll $a$ and $c$ were determined after $18-24$ hour cold extraction of the pigment in $90 \%$ acetone. All spectrophotometric readings were done using a Spectro UV-11 spectrophotometer. Dissolved oxygen DO values measured with the $\mathrm{Hy}-$ drolab were cross-checked with the Winkler titration method. Biochemical oxygen demand (BOD) was measured by the 5-day BOD test [23]. Chemical analyses were done at the Department of Biological Sciences, Masinde Muliro University of Science and Technology (MMUST), Kakamega, Kenya.

\subsection{Statistical Analysis}

Descriptive statistics (means and standard errors) for each treatment were computed using the data analysis pack on excel. QQ plots were used to check the normality of data whereas homogeneity of variance was assessed using Levene's test. A two way analysis of variance was used to compare means between treatments. Pairwise comparison of treatment was executed using Turkey's Honest Significant difference method. All statistical tests were conducted using R-statistical software version 3.6.1 with $95 \%$ confidence level.

\section{Results}

\subsection{Water Quality Characteristics of Sampling Sites}

A summary of water quality characteristics of the sampling sites is presented in 
Table 2 below. The mean $\mathrm{pH}$ values varied significantly with sites $(p<0.05)$ but were insignificantly variable with distance from lakeshore $(p>0.05)$. The highest $\mathrm{pH}$ values were $9.3 \pm 0.0$ and $9.3 \pm 0.1$ observed at Luanda Kotieno and Luanda Nyamasari respectively. The lowest $\mathrm{pH}$ values were $7.08 \pm 0.1$ and $7.08 \pm 0.0 \mathrm{ob}$ served in samples collected from deeper waters at Nyachebe and Kichinjio sites respectively. The mean temperature values observed varied significantly ( $p<$ $0.05)$ with site and distance from the lake. The highest mean temperature was $29.5^{\circ} \mathrm{C} \pm 0.0^{\circ} \mathrm{C}$ as observed at Luanda Nyamasari whereas the lowest mean temperature was $23.4^{\circ} \mathrm{C} \pm 0.2^{\circ} \mathrm{C}$ at Kichinjio.

The mean conductivity varied significantly $(p<0.05)$ with site and distance from the lake shore. The highest mean conductivity was $173.0 \pm 0.6 \mathrm{mS} \cdot \mathrm{m}^{-1}$ and as observed at Kichinjio, whereas the lowest was $69.0 \pm 1.7 \mathrm{mS} \cdot \mathrm{m}^{-1}$ as observed at Usenge. The mean TDS values also varied significantly $(p<0.05)$ with site and distance from the lakeshore. The highest mean TDS was $135.00 \pm 0.58 \mathrm{mg} \cdot \mathrm{l}^{-1}$ at Kagwel whereas the lowest mean TDS was $60.7 \pm 1.6 \mathrm{mg} \cdot \mathrm{l}^{-1}$ at Nyachebe. The mean DO values varied significantly $(p<0.05)$ with site and distance from the lakeshore too. The highest mean DO value was of $10.3 \pm 0.2 \mathrm{mg} \cdot \mathrm{l}^{-1}$ was observed at Luanda Kotieno whereas the lowest mean DO of $2.40 \pm 0.1 \mathrm{mg} \cdot \mathrm{l}^{-1}$ was recorded at Seka. Similarly, the mean BOD values varied significantly $(p<0.05)$ with site and distance from the lakeshore. The highest BOD mean value were 7.8 $\pm 0.3 \mathrm{mg} \cdot \mathrm{l}^{-1}$ recorded at Luanda Kotieno whereas the lowest BOD values were 0.0 $\pm 0.0 \mathrm{mg} \cdot \mathrm{l}^{-1}$ at Mainunga and Ngengu sites.

Mean turbidity values also varied significantly $(p<0.05)$ with sites and distance from the lakeshore. The highest mean turbidity value was $125.5 \pm 0.9 \mathrm{NTU}$ recorded at Uyoga whereas the lowest turbidity value was $2.7 \pm 0.1 \mathrm{NTU}$ observed at Osieko. The mean secchi depth readings too varied significantly $(p<$ $0.05)$ with site and distance from the lakeshore. The highest mean secchi readings were $1.2 \pm 0.0 \mathrm{~m}$ recorded at Nyachebe and Usenge. The lowest mean secchi value was $0.16 \pm 0.00 \mathrm{~m}$ as observed at Uyoga. Finally, same trend was recorded for mean TSS values which varied significantly with site $(p<0.05)$ and distance from the lakeshore. The highest mean TSS value was $10.2 \pm 0.3 \mathrm{mg} \cdot \mathrm{l}^{-1}$ and was observed at Nyamasari whereas the lowest was $0.3 \pm 0.1 \mathrm{mg} \cdot \mathrm{l}^{-1}$ was observed at Usenge. Generally, there was no a clear pattern observed between the values obtained in all the water quality parameters assessed and distance from the lakeshore.

\subsection{Nutrients and Chlorophyll Levels}

A summary of the nutrients and chlorophyll levels in samples collected from different sampling sites is presented in Table 3 below. Mean values of chlorophyll a (chl a) varied significantly with site $(p<0.05)$ and distance from the lakeshore. The highest mean $c h l$ a value was $66.9 \pm 0.9 \mu \mathrm{g} \cdot \mathrm{l}^{-1}$ and was observed at Kichinjio $(0 \mathrm{~m})$ whereas the lowest was $13.2 \pm 0.2 \mu \mathrm{g} \cdot \mathrm{l}^{-1}$ recorded at Seka. Mean chlorophyll $c(c h l c)$ values similarly varied significantly with site $(p<0.05)$ and distance from the lakeshore. The highest $c h l c$ mean of $134.7 \pm 3.8 \mu \mathrm{g} \cdot \mathrm{l}^{-1}$ was 
Table 2. Mean values ( \pm standard error) of water quality parameters measured in the study area.

\begin{tabular}{|c|c|c|c|c|c|c|c|c|c|c|}
\hline SITE & $\begin{array}{l}\text { Distance } \\
\text { from shore }\end{array}$ & $\mathrm{pH}$ & TEMP & COND & TDS & DO & BOD & TURB & SECC & TSS \\
\hline \multirow[t]{2}{*}{ Nyachebe } & $0-1 \mathrm{~m}$ & $7.7 \pm 0.11$ & $26.6 \pm 0.49$ & $86.5 \pm 0.55$ & $63.0 \pm 0.58$ & $4.5 \pm 0.06$ & $1.9 \pm 0.03$ & $41.0 \pm 1.15$ & $0.4 \pm 0.01$ & $6.1 \pm 0.26$ \\
\hline & $100 \mathrm{~m}$ & $7.1 \pm 0.02$ & $25.7 \pm 0.03$ & $80.0 \pm 2.08$ & $60.7 \pm 1.86$ & $4.3 \pm 0.10$ & $1.5 \pm 0.15$ & $3.6 \pm 0.15$ & $1.2 \pm 0.10$ & $7.2 \pm 0.17$ \\
\hline \multirow[t]{2}{*}{ Uyoga } & $0-1 \mathrm{~m}$ & $8.4 \pm 0.12$ & $26.9 \pm 0.06$ & $107.0 \pm 1.53$ & $73.0 \pm 1.54$ & $6.6 \pm 0.10$ & $2.9 \pm 0.06$ & $125.5 \pm 0.90$ & $0.16 \pm 0.00$ & $7.7 \pm 0.12$ \\
\hline & $100 \mathrm{~m}$ & $7.8 \pm 0.06$ & $25.1 \pm 0.07$ & $94.2 \pm 0.44$ & $65.9 \pm 0.61$ & $6.7 \pm 0.29$ & $4.0 \pm 0.45$ & $16.0 \pm 2.08$ & $0.7 \pm 0.06$ & $7.2 \pm 0.60$ \\
\hline \multirow[t]{2}{*}{$\begin{array}{c}\text { Luanda } \\
\text { Nyamasari }\end{array}$} & $0-1 \mathrm{~m}$ & $8.9 \pm 0.01$ & $26.7 \pm 0.65$ & $111.5 \pm 1.04$ & $71.9 \pm 1.01$ & $7.7 \pm 0.10$ & $5.0 \pm 1.00$ & $78.2 \pm 0.44$ & $0.2 \pm 0.02$ & $1.0 \pm 0.06$ \\
\hline & $100 \mathrm{~m}$ & $9.3 \pm 0.09$ & $29.5 \pm 0.00$ & $111.0 \pm 0.58$ & $78.0 \pm 0.76$ & $7.9 \pm 0.06$ & $3.5 \pm 0.25$ & $51.5 \pm 0.59$ & $0.36 \pm 0.00$ & $10.2 \pm 0.29$ \\
\hline \multirow[t]{2}{*}{ Ngengu } & $0-1 \mathrm{~m}$ & $8.1 \pm 0.09$ & $26.3 \pm 0.12$ & $134.5 \pm 0.81$ & $94.0 \pm 0.50$ & $4.2 \pm 0.10$ & $1.9 \pm 0.13$ & $43.6 \pm 0.79$ & $0.3 \pm 0.00$ & $7.4 \pm 0.20$ \\
\hline & $100 \mathrm{~m}$ & $8.6 \pm 0.07$ & $26.7 \pm 0.15$ & $119.0 \pm 1.15$ & $84.0 \pm 1.04$ & $5.4 \pm 0.12$ & $0.0 \pm 0.00$ & $63.2 \pm 1.13$ & $0.3 \pm 0.01$ & $2.5 \pm 0.44$ \\
\hline \multirow[t]{2}{*}{ Mainunga } & $0-1 \mathrm{~m}$ & $8.5 \pm 0.04$ & $27.0 \pm 0.58$ & $124.5 \pm 1.01$ & $86.0 \pm 0.12$ & $6.5 \pm 0.29$ & $2.4 \pm 0.06$ & $42.5 \pm 0.29$ & $0.4 \pm 0.01$ & $7.2 \pm 0.04$ \\
\hline & $100 \mathrm{~m}$ & $8.3 \pm 0.03$ & $26.3 \pm 0.12$ & $119.0 \pm 0.00$ & $83.0 \pm 0.58$ & $6.0 \pm 0.10$ & $0.0 \pm 0.00$ & $41.8 \pm 0.31$ & $0.4 \pm 0.00$ & $1.5 \pm 0.06$ \\
\hline Seka & $0-1 \mathrm{~m}$ & $7.3 \pm 0.01$ & $27.0 \pm 0.09$ & $126.5 \pm 0.00$ & $82.0 \pm 1.04$ & $2.4 \pm 0.06$ & $0.4 \pm 0.10$ & $48.3 \pm 0.15$ & $0.3 \pm 0.01$ & $6.7 \pm 0.05$ \\
\hline \multirow[t]{2}{*}{ Dunga } & $0-1 \mathrm{~m}$ & $7.8 \pm 0.01$ & $28.9 \pm 0.95$ & $139.0 \pm 1.53$ & $118.0 \pm 1.00$ & $8.3 \pm 0.07$ & $4.9 \pm 0.28$ & $53.2 \pm 0.12$ & $0.3 \pm 0.00$ & $4.6 \pm 0.06$ \\
\hline & $100 \mathrm{~m}$ & $8.0 \pm 0.03$ & $27.6 \pm 0.00$ & $123.0 \pm 0.29$ & $109.0 \pm 0.00$ & $7.2 \pm 0.03$ & $3.1 \pm 0.00$ & $48.4 \pm 0.00$ & $0.3 \pm 0.00$ & $1.4 \pm 0.08$ \\
\hline \multirow[t]{2}{*}{ Kichinjio } & $0-1 \mathrm{~m}$ & $7.8 \pm 0.15$ & $28.8 \pm 0.00$ & $173.0 \pm 0.58$ & 129.01 .15 & $5.0 \pm 0.03$ & $3.6 \pm 0.12$ & $69.5 \pm 0.00$ & $0.3 \pm 0.00$ & $5.0 \pm 0.58$ \\
\hline & $100 \mathrm{~m}$ & $7.1 \pm 0.02$ & $23.4 \pm 0.20$ & $169.0 \pm 1.13$ & $109.0 \pm 0.00$ & $4.0 \pm 0.17$ & $2.2 \pm 0.17$ & $70.5 \pm 0.00$ & $0.3 \pm 0.00$ & $3.2 \pm 0.15$ \\
\hline \multirow[t]{2}{*}{ Kagwel } & $0-1 \mathrm{~m}$ & $7.1 \pm 0.06$ & $27.3 \pm 1.97$ & $134.0 \pm 0.00$ & $135.0 \pm 0.58$ & $7.1 \pm 0.07$ & $4.7 \pm 0.12$ & $84.1 \pm 0.41$ & $0.3 \pm 0.00$ & $3.8 \pm 0.09$ \\
\hline & $100 \mathrm{~m}$ & $8.7 \pm 0.00$ & $27.3 \pm 0.88$ & $150.0 \pm 0.29$ & $116.0 \pm 0.00$ & $4.0 \pm 0.16$ & $1.5 \pm 0.15$ & $70.3 \pm 0.00$ & $0.3 \pm 0.01$ & $1.6 \pm 0.09$ \\
\hline \multirow[t]{2}{*}{$\begin{array}{l}\text { Luanda } \\
\text { Kotieno }\end{array}$} & $0-1 \mathrm{~m}$ & $9.3 \pm 0.03$ & $29.3 \pm 0.17$ & $87.0 \pm 0.07$ & $91.0 \pm 0.58$ & $10.3 \pm 0.22$ & $7.8 \pm 0.27$ & $12.3 \pm 0.76$ & $0.5 \pm 0.00$ & $2.6 \pm 0.15$ \\
\hline & $100 \mathrm{~m}$ & $8.3 \pm 0.73$ & $25.5 \pm 1.18$ & $95.0 \pm 0.73$ & $90.0 \pm 1.24$ & $5.5 \pm 0.07$ & $2.4 \pm 0.12$ & $13.3 \pm 0.44$ & $0.5 \pm 0.00$ & $0.4 \pm 0.06$ \\
\hline \multirow[t]{2}{*}{ Usenge } & $0-1 \mathrm{~m}$ & $7.3 \pm 0.25$ & $25.6 \pm 1.12$ & $76.0 \pm 0.04$ & $72.8 \pm 1.25$ & $3.9 \pm 0.46$ & $0.0 \pm 0.00$ & $4.0 \pm 0.20$ & $0.9 \pm 0.00$ & $0.3 \pm 0.05$ \\
\hline & $100 \mathrm{~m}$ & $8.1 \pm 0.58$ & $25.3 \pm 1.10$ & $69.0 \pm 1.73$ & $71.3 \pm 0.88$ & $5.2 \pm 1.38$ & $2.4 \pm 0.03$ & $3.5 \pm 0.00$ & $1.2 \pm 0.00$ & $3.2 \pm 0.06$ \\
\hline \multirow[t]{2}{*}{ Osieko } & $0-1 \mathrm{~m}$ & $7.8 \pm 0.01$ & $26.8 \pm 0.00$ & $80.0 \pm 2.89$ & $68.0 \pm 1.53$ & $4.1 \pm 0.44$ & $1.6 \pm 0.19$ & $5.2 \pm 0.15$ & $0.3 \pm 0.00$ & $1.3 \pm 0.12$ \\
\hline & $100 \mathrm{~m}$ & $7.8 \pm 0.03$ & $26.6 \pm 0.00$ & $73.0 \pm 2.89$ & $65.0 \pm 0.00$ & $5.4 \pm 0.44$ & $2.7 \pm 0.33$ & $2.7 \pm 0.06$ & $0.8 \pm 0.00$ & $0.8 \pm 0.15$ \\
\hline
\end{tabular}

TEMP $=$ temperature, $\mathrm{COND}=$ conductivity, $\mathrm{TDS}=$ total dissolved solids, $\mathrm{DO}=$ dissolved oxygen, $\mathrm{BOD}=$ biochemical oxygen demand, TURB $=$ turbidity, SECC $=$ secchi depth and TSS $=$ total suspended solids. Units: $\mathrm{pH}\left(\mathrm{pH}\right.$ units), temperature $\left({ }^{\circ} \mathrm{C}\right)$, conductivity $\left(\mathrm{mS} \cdot \mathrm{m}^{-1}\right)$, TDS $\left(\mathrm{mg} \cdot \mathrm{l}^{-1}\right), \mathrm{DO}\left(\mathrm{mg}^{\circ} \cdot \mathrm{O}_{2} \cdot \mathrm{l}^{-1}\right), \mathrm{BOD} \mathrm{D}_{5}$ $\left(\mathrm{mg} \cdot \mathrm{l}^{-1}\right)$, turbidity $\left(\mathrm{NTU}=\right.$ Nephelometric turbidity units), Secchi depth $(\mathrm{m})$, TSS $\left(\mathrm{mg} \cdot \mathrm{l}^{-1}\right)$.

recorded at Luanda Kotieno whereas the lowest $\left(0.63 \pm 0.1 \mu \mathrm{g} \cdot \mathrm{l}^{-1}\right)$ was recorded at Kagwel. Similar trend was observed for mean $\mathrm{PO}_{4}-\mathrm{P}$ values obtained from the analysis which varied significantly with site $(p<0.05)$ and distance from the lakeshore too. The highest mean $\mathrm{PO}_{4}-\mathrm{P}$ was $310.7 \pm 1.7 \mu \mathrm{g} \cdot \mathrm{l}^{-1}$ observed at Dunga whereas Luanda Kotieno posted the lowest $\mathrm{PO}_{4}-\mathrm{P}$ of $28.9 \pm 1.4 \mu \mathrm{g} \cdot \mathrm{l}^{-1}$. Dunga again recorded the highest mean $\mathrm{NH}_{4}-\mathrm{N}$ of $1278.3 \pm 6.7 \mu \mathrm{g} \cdot \mathrm{l}^{-1}$ whereas Luanda Nyamasarire corded the least $\left(12.4 \pm 0.8 \mu \mathrm{g} \cdot \mathrm{l}^{-1}\right)$.

\section{Discussion}

Freshwater ecosystems, such as lakes, wetlands and rivers have specific requirements in terms of quantity, quality and seasonality of their water supplies. 
Table 3. Mean ( \pm standard error) of nutrients and chlorophyll levels in samples collected from different sites in the study area.

\begin{tabular}{|c|c|c|c|c|c|}
\hline SITE & Distance from shore & Chla & Chlc & $\mathrm{PO}_{4}-\mathrm{P}$ & $\mathrm{NH}_{4}-\mathrm{N}$ \\
\hline \multirow[t]{2}{*}{ Nyachebe } & $0 \mathrm{~m}$ & $34.8 \pm 0.09$ & $15.2 \pm 0.49$ & $111.2 \pm 0.55$ & $322.3 \pm 6.78$ \\
\hline & $100 \mathrm{~m}$ & $17.2 \pm 0.38$ & $28.3 \pm 1.39$ & $49.1 \pm 1.48$ & $19.0 \pm 1.49$ \\
\hline \multirow[t]{2}{*}{ Uyoga } & $0 \mathrm{~m}$ & $27.2 \pm 0.73$ & $79.7 \pm 0.93$ & $84.3 \pm 0.67$ & $35.8 \pm 1.97$ \\
\hline & $100 \mathrm{~m}$ & $26.5 \pm 1.33$ & $78.2 \pm 1.30$ & $57.5 \pm 3.16$ & $67.2 \pm 2.57$ \\
\hline \multirow[t]{2}{*}{ L. Nyamasari } & $0 \mathrm{~m}$ & $15.1 \pm 0.82$ & $22.5 \pm 0.64$ & $69.2 \pm 1.42$ & $15.4 \pm 0.90$ \\
\hline & $100 \mathrm{~m}$ & $62.9 \pm 1.08$ & $89.1 \pm 0.47$ & $94.4 \pm 1.29$ & $12.4 \pm 0.79$ \\
\hline \multirow[t]{2}{*}{ Ngengu } & $0 \mathrm{~m}$ & $16.4 \pm 0.87$ & $17.6 \pm 0.34$ & $65.7 \pm 0.88$ & $714.1 \pm 6.45$ \\
\hline & $100 \mathrm{~m}$ & $64.2 \pm 0.82$ & $35.5 \pm 1.27$ & $84.2 \pm 0.42$ & $108.2 \pm 1.33$ \\
\hline \multirow[t]{2}{*}{ Mainunga } & $0 \mathrm{~m}$ & $27.3 \pm 0.44$ & $97.4 \pm 0.50$ & $55.8 \pm 0.76$ & $45.3 \pm 0.57$ \\
\hline & $100 \mathrm{~m}$ & $27.0 \pm 1.15$ & $92.2 \pm 0.4$ & $102.8 \pm 0.20$ & $51.2 \pm 0.67$ \\
\hline Seka & $0 \mathrm{~m}$ & $13.2 \pm 0.15$ & $44.7 \pm 0.44$ & $96.1 \pm 0.46$ & $259.5 \pm 2.02$ \\
\hline \multirow[t]{2}{*}{ Dunga } & $0 \mathrm{~m}$ & $25.5 \pm 0.29$ & $94.4 \pm 0.81$ & $310.7 \pm 1.70$ & $1278.3 \pm 6.61$ \\
\hline & $100 \mathrm{~m}$ & $42.8 \pm 0.64$ & $110.0 \pm 1.53$ & $107.8 \pm 1.18$ & $182.7 \pm 0.77$ \\
\hline \multirow[t]{2}{*}{ Kichinjio } & $0 \mathrm{~m}$ & $28.4 \pm 0.23$ & $5.1 \pm 0.06$ & $111.2 \pm 1.92$ & $336.9 \pm 1.02$ \\
\hline & $100 \mathrm{~m}$ & $66.9 \pm 0.97$ & $77.0 \pm 0.58$ & $109.5 \pm 0.50$ & $331.1 \pm 1.16$ \\
\hline \multirow[t]{2}{*}{ Kagwel } & $0 \mathrm{~m}$ & $31.5 \pm 0.58$ & $0.6 \pm 0.09$ & $114.5 \pm 0.62$ & $244.9 \pm 3.79$ \\
\hline & $100 \mathrm{~m}$ & $20.4 \pm 0.67$ & $75.7 \pm 0.88$ & $111.2 \pm 0.95$ & $100.1 \pm 1.55$ \\
\hline \multirow[t]{2}{*}{ L. Kotieno } & $0 \mathrm{~m}$ & $30.1 \pm 1.16$ & $119.9 \pm 1.16$ & $29.0 \pm 1.39$ & $192.1 \pm 0.99$ \\
\hline & $100 \mathrm{~m}$ & $22.9 \pm 1.48$ & $134.7 \pm 3.81$ & $40.0 \pm 1.15$ & $251.1 \pm 0.25$ \\
\hline \multirow[t]{2}{*}{ Usenge } & $0 \mathrm{~m}$ & $22.6 \pm 1.30$ & $101.4 \pm 1.52$ & $60.9 \pm 0.65$ & $168.1 \pm 2.04$ \\
\hline & $100 \mathrm{~m}$ & $35.1 \pm 0.64$ & $76.4 \pm 1.81$ & $49.1 \pm 2.45$ & $145.5 \pm 1.85$ \\
\hline \multirow[t]{2}{*}{ Osieko } & $0 \mathrm{~m}$ & $18.5 \pm 0.70$ & $73.5 \pm 1.32$ & $54.1 \pm 2.03$ & $99.4 \pm 02.27$ \\
\hline & $100 \mathrm{~m}$ & $17.4 \pm 0.30$ & $103.7 \pm 1.20$ & $70.9 \pm 2.05$ & $63.6 \pm 0.75$ \\
\hline
\end{tabular}

Units: Chlorophyll a $\left(\mu \mathrm{g} \cdot \mathrm{l}^{-1}\right)$, Chlorophyll c $\left(\mu \mathrm{g} \cdot \mathrm{l}^{-1}\right)$, Phosphate phosphorous $\left(\mathrm{PO}_{4}-\mathrm{P}\right) \mu \mathrm{g} \cdot \mathrm{l}^{-1}$, Ammoniacal nitrogen $\left(\mathrm{NH}_{4}-\mathrm{N}\right) \mu \mathrm{g} \cdot \mathrm{l}^{-1}$.

Sustainability normally requires these systems to fluctuate within a natural range of variation. One of the fundamental factors that are affected by water pollution is the $\mathrm{pH}$. Overally, the $\mathrm{pH}$ values observed in the current study had a higher upper limit compared to earlier findings [24] though all sampling sites with an exception of Kagwel and Nyamasari posted $\mathrm{pH}$ values that were still within the optimal range (6.5 - 9.0) for most aquatic chemical reactions as well as aquatic organisms' life including fish. It is worth noting that $\mathrm{pH}$ values outside the optimal range would have negative implications on aquatic life such as increased toxicity of chemical compounds, reduced hatching and survival rates among others [25]. The variations of $\mathrm{pH}$ values observed among the sampling sites could be an indication of the different levels of respiration and decomposition activities taking place below the lake surface among the different sampling sites, 
and varying volumes of wastewater discharged [26].

Water temperature influences several processes in aquatic environment including metabolic rates, photosynthesis production as well as levels of other water quality parameters such as dissolved gases, $\mathrm{pH}$ and conductivity. Current study showed temperature variations across sampling sites and this could be associated with varying loads of total suspended solids discharged by rivers and domestic runoffs into the lake which in turn absorb solar energy hence increasing temperature [24]. Generally, the sites that reported higher temperatures also posted high $\mathrm{pH}$ values. [27], noted that as temperature increases or decreases, a shift in ion concentrations in the water changes thus affecting the $\mathrm{pH}$ value. Therefore, the higher temperature directly affected the $\mathrm{pH}$ through dissociation and solubility of certain compounds in the water column [28].

The toxicity of ammonia $\left(\mathrm{NH}_{3}\right)$ is critically dependent on $\mathrm{pH}$ and temperature. As $\mathrm{pH}$ increases, ammonium cation $\left(\mathrm{NH}_{4}^{+}\right)$is converted to $\mathrm{NH}_{3}$, and the toxicity increases [29]. In the current study, ammoniacal nitrogen was highest at Luanda Nyamasari an occurrence that is closely linked to the sites high temperature and $\mathrm{pH}$. Higher nutrients levels in the gulf have also been reported in earlier studies by [24] [30]. This could be an indication that there was municipal, agricultural and industrial pollution in these sites since nutrients and sediments from far inland enter the lake directly without being filtered as a result of clearing of vegetation surrounding the Lake. [31] noted that fishing shoreline settlements have less than $20 \%$ of pit latrines in place thus most human waste is discharged directly into L. Victoria. This nitrogen would equally be flux from the sediments to the water column in most of the landing sites such as Ngegu shoreline [32]. Buildup of aquatic macrophytes including algae and hyacinth contribute significantly to higher $\mathrm{NH}_{3}$ levels as they die off and are swept by waves to decay in sheltered shores of the lake.

Extensive water hyacinth mats frequently settle in near-shore waters for considerable periods of time. When this is tied to the removal of $\mathrm{NO}_{3}$ efficiency of the lake, it should be noted that the higher $\mathrm{NO}_{3}$ removal is as a result of uptake by the dominant phytoplankton species which is reflected by the total dissolved solids, EC and secchi depth readings. Low nutrient removal efficiency even under conditions of high nutrient concentrations could be light limitation of phytoplankton growth due to excessive chlorophyll a, an occurrence witnessed in this study. The highest chl a value, $66.9 \mu \mathrm{g} \cdot \mathrm{l}^{-1}$ was recorded at Kichinjio where there was an apparent algal bloom on the water surface. The lowest chl a value, 13.2 $\mu \mathrm{g} \cdot \mathrm{l}^{-1}$ was recorded at Mainunga a site limited in human activity, runoff and aquatic vegetation degradation compared to high density human population at Kichinjio. The concentration of chl a fluctuated irregularly across the sampling sites although slightly high values occurred in mid Nyanza Gulf sites. Higher values in sites inside the lake indicated larger amounts of photosynthesizing phytoplankton or algae compared to the shoreline. chl $c$ also fluctuated rather irregularly with the highest value being $134.7 \mu \mathrm{g} \cdot \mathrm{l}^{-1}$ at Luanda Kotienoan occurrence closely linked to the sites elevated $\mathrm{NH}_{4}-\mathrm{N}$ at $100 \mathrm{~m}$ offshore compared to 
all the other sites.

Dissolved oxygen is critical for key biological processes such as decomposition and respiration in plants, animals and bacteria in aquatic systems. Aquatic organisms DO requirements vary widely and are also tied to the organisms' vertical and horizontal trophic position. In the current study, DO fluctuated between 2.0 and $10.0 \mathrm{mg} / \mathrm{L}$ across the different sampling sites, however there was no clear pattern with respect to distance from the shore a factor of lake water currents, river inflows, algal blooms and suspended solids among others as has also been previously reported [33] [34].

Total Suspended Solids (TSS) are organic and inorganic solid materials that are suspended in water. They include silt, plankton and industrial wastes which will not pass through the filter [35]. Findings from the study in L. Victoria Kenya side had turbidity and TSS increase at the shoreline in most of the sampling sites an indicator of inputs of soil and sediments from the catchments mainly through runoff, shoreline erosion, and re-suspension of bottom sediments by waves, currents, lake mixing as well as human related activities. Whereas, phytoplankton is the main contributor of offshore turbidity and TSS, secchi depth was inverse to the levels of these two variables since it largely depended on the water clarity. The highest secchi depth values recorded at Nyachebe and Usenge off shore were a factor of the sites exposure to north western and southern parts of the open lake. The turbidity values observed in most of sampling sites in the current study were much lower compared to an earlier [24] perhaps due to the differences in locations of sampling sites. Turbidity and TSS though related did not demonstrate any correlation within and across sampling sites.

Pollution is viewed as a serious threat to water quality for its direct and indirect impacts on lake communities [36]. Silt, clay and other organic particles can suffocate larvae and clog gills of many aquatic organisms and interfere with their normal functioning or even cause death. They can also interfere with light penetration into the water column retarding growth of phytoplankton and macrophytes thereby lowering primary productivity, release of oxygen into the water and life of other aquatic organisms. Silt, clay and other organic particles are also known to form attachment substrates for many types of bacteria and other micro-organisms. The WHO [37] and EPA [28] standards for turbidity of drinking water is a value of less than 5 Nephelometric Turbidity Units (NTU) and appear clear [38]. In this study, turbidity values were generally higher in sites of the mid Nyanza Gulf and decreased irregularly towards the north western and Southern sites all exposed to open lake dilution effect. Majority of the high values were recorded near the shoreline than deeper parts of the lake. Similarly, higher values of TSS were recorded in the southern sites and reduced towards the north.

Total dissolved solids (TDS) refer to all ion particles in solution that are smaller than two microns [39]. It is a measure of the combined content of all organic and inorganic substances contained in a liquid in molecular, ionized or microgranular suspended form. The primary sources of TDS are the agricultural and residential runoff, leaching of soil contamination and point source water 
pollution discharge from industrial and sewerage treatment plants. TDS majorly constitutes the ions; sulphates $\left(\mathrm{SO}^{3-}\right), \mathrm{Cl}^{-}$, carbonates $\left(\mathrm{CO}^{3-}\right)$, and cations; potassium $\left(\mathrm{K}^{+}\right)$, Sodium $\left(\mathrm{Na}^{+}\right)$, magnesium $\left(\mathrm{Mg}^{2+}\right)$ and calcium $\left(\mathrm{Ca}^{2+}\right)$. Like conductivity, the levels of TDS are influenced by dissolved ions and salts, in addition to micro-colloidal substances. The TDS were below $500 \mathrm{mg} / \mathrm{l}$ thus were within the acceptable levels according to [28].

Rain washes sediments and nutrients from bare soil into receiving waters [32]. Sediment analysis showed that nitrogen flux from the sediments to the water column is more pronounced than the phosphorus flux in most of the landing sites. This suggests that the phosphorus loading to the lake may be primarily external rather than from the sediments. Continuous mixing of the Lake waters by the wind, especially during late afternoons and early morning hours when winds are strongest in the gulf [40] [41] helped to keep the gulf well oxygenated throughout the year and led to the re-suspension of bottom sediments, which contributed to low water transparency in the gulf. Elevated phosphates near the shoreline waters are due to inputs resulting from weathering processes of the substratum and land erosion from the almost bare or cultivated land and other anthropogenic activities, mineralization of organic matter and recycling from decomposing dead water hyacinth plants. Seka which had the highest $\mathrm{PO}_{4}-\mathrm{P}$ had a black muddy substrate and decomposing water hyacinth plants. The presence of readily measured $\mathrm{PO}_{4}-\mathrm{P}$ and $\mathrm{NO}_{3}-\mathrm{N}$ concentrations throughout the gulf and the inverse relation between Secchi disc depth and chl a is consistent with light limitation of algal growth and nutrient demand. The increased release of phosphorus from the sediment that occurred during dry season was associated with elevated temperature and $\mathrm{pH}$ [42] [43] and inversely lower DO concentration thus promoting anaerobic condition.

\section{Conclusion and Recommendations}

In the current study, water quality parameters varied significantly across the different sampling sites in the area studied. Extreme values of different water quality parameters (DO, $\mathrm{pH}$, turbidity and nutrients) observed in different sites could be an indication that the onshore activities in the different study sites are altering the water quality of the study area. Values outside the recommended ranges in some of the water quality parameters in the current study were posted both in nearshore and offshore sampling site, an indicator that pollution in the study area is not restricted to lakeshore but also offshore probably due to adequate mixing of water in the study area. It is worth noting that much higher values of temperature, $\mathrm{pH}$ and ammoniacal nitrogen were posted in the current study compared to earlier studies conducted in the study area which could be an indicator of progressive deterioration of the water quality thus the need to review the management measures and interventions in place. Further studies of this kind more so linking fish populations and catch statistics with water quality and onshore human activities would provide important information for comprehensive sustainable management plan of the resource. 


\section{Conflicts of Interest}

The authors declare no conflict of interest regarding the publication of this paper.

\section{References}

[1] LVEMP III (2018) Project Information Document/Integrated Safeguards Data Sheet (PID/ISDS).

[2] Njiru, M., Kazungu, J., Ngugi, C., Gichuki, J. and Muhhozi, L. (2008) An Overview of the Current Status of Lake Victoria Fishery: Opportunities, Challenges and Management Strategies. Lakes \& Reservoirs: Research and Management, 13, 1-12. https://doi.org/10.1111/j.1440-1770.2007.00358.x

[3] Gikuma-Njuru, P. (2012) An Overview of the Present Status of Water Quality of Lake Victoria, Kenya: A Limnological Perspective.

[4] Swedish International Development Cooperation Agency (2007) Lake Victoria. A Shared Vision.

https://www.sida.se/contentassets/b744a361f9d644289bb15f445f29e414/lakevictoria -asharedvision_1325.pdf

[5] Baron, J.S., Poff, N.L., Angermeier, P.L., Dahmm, C.N., Gleick, P.H., Hairston, N.G., Jackson, R.B., Johnston, C.A., Richter, B.D. and Steinman, A.D. (2002) Meeting Ecological and Societal Needs for Freshwater. Ecological Applications, 12, 1247-1260. https://doi.org/10.1890/1051-0761(2002)012[1247:MEASNF]2.0.CO;2

[6] Subhankar, K. and Musthafa, O.M. (2013) Lakes and Reservoirs: Pollution. In: Encyclopedia of Environmental Management, Taylor and Francis, New York, 1576-1587.

[7] Ochumba, P.B.O. and Kibaara, D.I. (1989) Observations on Blue-Green Algal Blooms in the Open Waters of Lake Victoria, Kenya. African Journal of Ecology, 27, 23-34. https://doi.org/10.1111/j.1365-2028.1989.tb00925.x

[8] Gikuma-Njuru, P., Guildford, S., Hecky, R. and Kling, H. (2013) Strong Spatial Differentiation of $\mathrm{N}$ and P Deficiency, Primary Productivity and Community Composition between Nyanza Gulf and Lake Victoria (Kenya, East Africa) and the Implications for Nutrient Management. Freshwater Biology, 58, 2237-2252. https://doi.org/10.1111/fwb.12205

[9] Kansiime, F., Kateyo, E. and Okot-Okumu, J. (1995) Effects of Pollution of Inner Murchison Bay (Lake Victoria, Uganda) on the Distribution and Abundance of Plankton. NUFU Report, Faculty of Science, Makerere University, Kampala, 30 p.

[10] Oguttu, H.W., Bugenyi, F.W.B., Leuenberger, H., Wolf, M. and Bachofen, R. (2008) Pollution Menacing Lake Victoria: Quantification of Point Sources around Jinja Town, Uganda. Water $S A, 34,89-98$. https://doi.org/10.4314/wsa.v34i1.180865

[11] Onyango, A.A., Lalah, J.O., Wandiga, S.O. and Gichuki, J. (2012) Assessment of Polycyclic Aromatic Hydrocarbons in Lates niloticus, Oreochromis niloticus and Rastrineobolaargenteaas Sources of Human Exposure in Kisumu Bay, Winam Gulf of Lake Victoria. Bulletin of Environmental Contamination and Toxicology, 88, 747-751. https://doi.org/10.1007/s00128-012-0567-1

[12] Omwoma, S., Lalah, J.O., Virani, M., Schramm, K. and Henkelmann, B. (2015) Dioxin-Like PCBs and PCDD/Fs in Surface Sediments near the Shore of Winam Gulf, Lake Victoria. Chemosphere, 118, 143-147. https://doi.org/10.1016/j.chemosphere.2014.07.062

[13] Moses, A.N., Nyongesa, N., Destaings, N.E.M. and Miima, J. (2011) Effluent Dis- 
charge by Mumias Sugar Company in Kenya: An Empirical Investigation of the Pollution of River Nzoia. Sacha Journal of Environmental Studies, 1, 1-301.

[14] UNESCO-IIWQ (2015) Emerging Pollutants in Wastewater Reuse in Developing Countries. Case Studies on Emerging Pollutants in Water and Wastewater. 103.

[15] Bongomin, J. (2011) Modeling Non-Point Source Pollution in Lake Victoria: A Case of Gaba Landing Site. 32-48.

[16] Rosegrant, M.W., Cai, X., Cline, S.A. and Nakagawa, N. (2002) The Role of Rainfed Agriculture in the Future of Global Food Production (No. 581-2016-39482).

[17] Statista (2019) Projected Increase in Global Annual Water Demand from 2005 to 2030, by Region and Sector (in Billion Cubic Meters). https://www.statista.com/statistics/278066/global-water-demand-by-region

[18] UNEP (2011) UNEP Annual Report 2010. United Nations Environment Programme, Nairobi.

[19] Jackson, M., Loewen, C., Vinebrooke, D. and Chimimba, T. (2016) Net Effects of Multiple Stressors in Freshwater Ecosystems: A Meta-Analysis. Global Change Biology, 22, 180-189. https://doi.org/10.1111/gcb.13028

[20] Carpenter, S.R., Kitchel, S.F. and Hodgson, J.F. (1985) Cascading Trophic Interactions and Ake Productivity. Bioscience, 35, 634-639.

https://doi.org/10.2307/1309989

[21] Schindler, D.E., Geib, S.I. and Williams, M.R. (2000) Patterns of Fish Growth along a Residential Development Gradient in North Temperate Lakes. Ecosystems, 3, 229-237. https://doi.org/10.1007/s100210000022

[22] American Public Health Association (APHA) (1995) Standard Methods for Analysis of Water and Wastewater. 18th Edition, Port City Press, Baltimore.

[23] Delzer, G.C. and McKenzie, S.W. (2003) Five-Day Biochemical Oxygen Demand. USGS, Reston.

[24] Nyakundi, J.K., Njiru, J.M., Mwayuli, G.A. and Olilo, C. (2015) Effects of Point Source Pollution on Water Quality, Phytoplankton Diversity and Abundance in Lake Victoria, Kenya. International Journal of Farming and Allied Sciences, 2, 57-64.

[25] Fondriest Environmental, Inc. (2013) “pH of Water” Fundamentals of Environmental Measurements.

http://www.fondriest.com/environmental-measurements/parameters/water-quality/ $\mathrm{pH}$

[26] Missouri, D.N.R. (2009) Stream Discharge. In: Introduction Level Volunteer Water Quality Monitoring Training Notebook, US Government, Washington DC, Chapter 3.

[27] Crone, T. (2004) The Basic Sediment Transport Equations Made Ridiculously Simple. In: Ocean/Ess 410 Marine Geology and Geophysics. http://www.ocean.washington.edu/courses/oc410/reading/sedtrans_2004

[28] Environmental Protection Agency (EPA) (2012) Total Dissolved Solids. In Water: Monitoring and Assessment. http://water.epa.gov/type/rsl/monitoring/vms58.cfm

[29] Emerson, K., Russo, R.C., Lund, R.E. and Thurston, R.V. (1975) Aqueous Ammonia Equilibrium Calculations: Effect of $\mathrm{pH}$ and Temperature. Journal of the Fisheries Board of Canada, 32, 2379-2383. https://doi.org/10.1139/f75-274

[30] Gikuma-Njuru, P. and Hecky, R.E. (2005) Nutrient Concentrations in Nyanza Gulf, Lake Victoria, Kenya: Light Limits Algal Demand and Abundance. Hydrobiologia, 534, 131-140. https://doi.org/10.1007/s10750-004-1418-9 
[31] Tonderski, A. (1996) Landuse-Based Nonpoint Source Pollution. A Threat to Water Resources in Developing Countries. Water Science and Technology, 33, 53-61. https://doi.org/10.2166/wst.1996.0488

[32] Livingston, H. and Borja, A. (1985) A Review of Urban Storm Water Retrofitting in Florida. Florida Department of Environmental Protection, Florida.

[33] Ochumba, P.B.O. (1990) Massive Fish Kills within Nyanza Gulf of Lake Victoria, Kenya. Hydrobiologia, 208, 93-99. https://doi.org/10.1007/BF00008448

[34] Hecky, R.E., Bugenyi, F.W.B., Ochumba, P.B.O., Talling, J.F., Mugidde, R., Gophen, M. and Kaufman, L. (1994) Deoxygenation of the Deep Water of Lake Victoria, East Africa. Limnology Oceanography, 39, 23-36. https://doi.org/10.4319/lo.1994.39.6.1476

[35] Fondriest Environmental, Inc. (2014) Turbidity, Total Suspended Solids and Water Clarity. Fundamentals of Environmental Measurements. https://www.fondriest.com/environmental-measurements/parameters/water-quality /turbidity-total-suspended-solids-water-clarity/

[36] Aaike, D.W., Koenraad, M., Denis, L., Laurent, A., Jean, D., Luc, A., Christine, C. and Wim, V. (2008) Differential Response of Phytoplankton to Additions of Nitrogen, Phosphorus and Iron in Lake Tanganyika. Journal of Freshwater Biology, 53, 264-277.

[37] WHO (2006) Guidelines of Drinking Water Quality.

[38] Ouma, S.O. (2015) Physico-Chemical and Bacteriological Quality of Water from Five Rural Catchment Areas of Lake Victoria Basin in Kenya. MSc Thesis, 8-22.

[39] Pawlowicz, R. (2013) TEOS-10 Primer, What Every Oceanographer Needs to Know about TEOS-10. In Thermodynamic Equation of Sea Water 2010.

http://www.teos-10.org/publications.htm

[40] Worthington, E.B. (1930) Observations on the Temperature, Hydrogen-Ion Concentration, and Other Physical Conditions of the Victoria and Albert Nyanzas. Inernationale Revue der Gesamten Hydrobiologie Und Hydrography, 24, 328-357. https://doi.org/10.1002/iroh.19300240306

[41] Njuru, P.G. (2001) An Overview of the Present Status of Water Quality of Lake Victoria, Kenya: A Limnological Perspective. Proceedings of Lake Victoria Environment Management Programme (LVEMP) First Regional Scientific Conference, Kisumu, 3-7 December 2001, 11.

[42] Cerco, C.F. (1989) Measured and Modelled Effects of Temperature, Dissolved Oxygen and Nutrient Concentration on Sediment-Water Nutrient Exchange. Hydrobiologia, 174, 185-194. https://doi.org/10.1007/BF00008156

[43] Irshad, M., Malik, N., Khan, T. and Faridullah, A. (2011) Effect of Solid Waste on Heavy Metal Composition of Soil and Water at Nathiagali-Abbottabad. Journal of the Chemical Society of Pakistan, 33, 830-834. 